\title{
Magnetic Circular Dichroism (MCD) Responses with High Sensitivity and Enhanced Spectral Resolution in Multipolar Plasmonic Modes of Silver Nanoparticles with Dimensions between 90 and 200 nm
}

\author{
Yuki Nagumo and Hiroshi Yao*
}

Division of Chemistry for Materials, Graduate School of Engineering, Mie University, 1577 Kurimamachiya-cho, Tsu, Mie 514-8507, Japan

Extinction and MCD spectra of Ag nanoparticles with the mean diameter of $60.5 \mathbf{~ n m}$.

Figure S1 shows UV-vis extinction and MCD spectra of the Ag nanoparticles $(60.5 \mathrm{~nm})$ in aqueous solution. MCD spectra were obtained under an applied magnetic field of $+1.6 \mathrm{~T}$ (blue curve) and $-1.6 \mathrm{~T}$ (red curve).

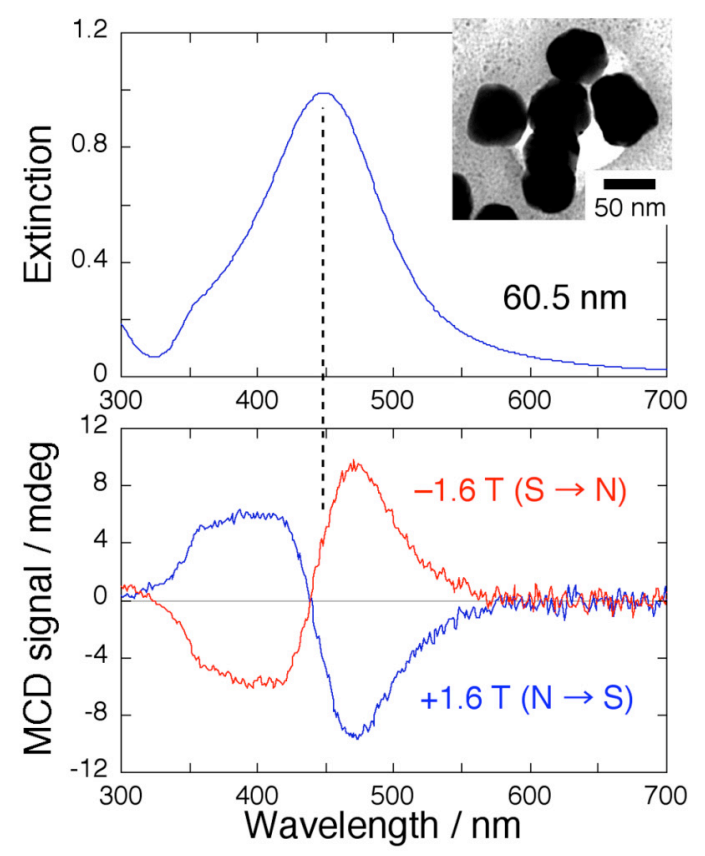

Figure S1. Extinction (upper) and MCD (lower) spectra of the Ag nanoparticle sample with the mean diameter of $60.5 \mathrm{~nm}$. The inset is a typical TEM image of the sample. 